,

\title{
Examination of the Nature of the ABC Effect
}

\author{
M. Bashkanov*, ${ }^{1}$ H. Clement, ${ }^{2,3}$ and T. Skorodko ${ }^{2,3,4}$ \\ ${ }^{1}$ School of Physics and Astronomy, University of Edinburgh, James Clerk \\ Maxwell Building, Peter Guthrie Tait Road, Edinburgh, EH9 3FD, UK \\ ${ }^{2}$ Physikalisches Institut, Eberhard-Karls-Universität Tübingen, Auf der Morgenstelle 14, D-72076 Tübingen, Germany \\ ${ }^{3}$ Kepler Center for Astro and Particle Physics, University of Tübingen, Auf der Morgenstelle 14, D-72076 Tübingen, Germany \\ ${ }^{4}$ Department of Physics, Tomsk State University, 36 Lenina Avenue, Tomsk 634050, Russia
}

(Dated: April 28, 2018)

\begin{abstract}
Recently it has been shown by exclusive and kinematically complete experiments that the appearance of a narrow resonance structure in double-pionic fusion reactions is strictly correlated with the appearance of the so-called ABC effect, which denotes a pronounced low-mass enhancement in the $\pi \pi$-invariant mass spectrum. Whereas the resonance structure got its explanation by the $d^{*}(2380)$ dibaryonic resonance, a satisfactory explanation for the $\mathrm{ABC}$ effect is still pending. In this paper we discuss possible explanations of the $\mathrm{ABC}$ effect and their consequences for the internal structure of the $d^{*}$ dibaryon. To this end we examine and review a variety of proposed explanations for the $\mathrm{ABC}$ effect, add a new hypothesis and confront all of them with the experimental results for the $n p \rightarrow d \pi^{0} \pi^{0}$ and $n p \rightarrow n p \pi^{0} \pi^{0}$ reactions, which are the most challenging ones for this topic.
\end{abstract}

PACS numbers: $13.75 . \mathrm{Cs}, 14.20 . \mathrm{Gk}, 14.20 . \mathrm{Pt}$

Keywords:

\section{INTRODUCTION}

Recently it was shown that there is a resonance pole at $(2380 \pm 10)-i(40 \pm 5) \mathrm{MeV}$ in the ${ }^{3} D_{3}-{ }^{3} G_{3}$ coupled partial waves based on a SAID partial-wave analysis, which included new data from COSY on the analyzing power of $n p$ scattering [1-4]. This finding matches perfectly with the $I\left(J^{P}\right)=0\left(3^{+}\right)$resonance structure observed at $\sqrt{s} \approx$ $2.37 \mathrm{GeV}$ with a width of $70 \mathrm{MeV}$ in the total cross section of the double-pionic fusion reactions $p n \rightarrow d \pi^{0} \pi^{0}$ and $p n \rightarrow d \pi^{+} \pi^{-}[5-7]$. The results from the WASAat-COSY for the $p n \rightarrow d \pi^{+} \pi^{-}$reaction have meanwhile found support by preliminary results from HADES [8].

Having revealed the pole in the $n p$ scattering amplitudes means that this resonance structure constitutes an $s$-channel resonance in the system of two baryons. It has been denoted since then by $d^{*}(2380)$ following the nomenclature used for nucleon excitations.

Follow-up measurements of the non-fusion reactions $p n \rightarrow p p \pi^{0} \pi^{-}[\underline{9}]$ and $p n \rightarrow p n \pi^{0} \pi^{0}[\underline{10}]$ with the WASA detector at COSY and $n p \rightarrow n p \pi^{+} \pi^{-}$[11] with the HADES detector at GSI showed that also these reactions, which are partially of isoscalar character, show the $d^{*}(2380)$ resonance in agreement with expectations based on isospin coupling.

In addition, WASA measurements revealed that $d^{*}(2380)$ is also present in the double-pionic fusion reactions to the helium isotopes $p d \rightarrow{ }^{3} \mathrm{He} \pi^{0} \pi^{0}$,

\footnotetext{
*corresponding author

email address: mikhail.bashkanov@ed.ac.uk
}

$p d \rightarrow{ }^{3} \mathrm{He} \pi^{+} \pi^{-}, d d \rightarrow{ }^{4} \mathrm{He} \pi^{0} \pi^{0}$ and $d d \rightarrow{ }^{4} \mathrm{He} \pi^{+} \pi^{-}$ 15. This means that obviously $d^{*}(2380)$ is stable enough to survive also in a nuclear surrounding. This conclusion is in agreement with the appearance of a dilepton enhancement (DLS puzzle) in heavy-ion collisions [16].

\section{ABC EFFECT}

In 1960 Abashian, Booth and Crowe 17] found out that the $\pi \pi$-invariant mass spectrum in the double-pionic fusion reaction $p d \rightarrow{ }^{3} \mathrm{He} \pi \pi$ exhibits a pronounced lowmass enhancement. Subsequent measurements showed that this enhancement also persists in the fusion reactions to $d$ and ${ }^{4} \mathrm{He}$ [18 28], if the produced pion pair is of isoscalar nature. Since there was no plausible explanation for this effect, it got named after the initials of the authors of the first publication on that issue.

The recent exclusive and kinematically complete WASA measurements, which were carried out at CELSIUS and later-on at COSY, confirmed the previous findings, which had been obtained by merely inclusive singlearm magnetic spectrometer and low-statistics bubblechamber measurements, respectively. Fig. 1 shows as an example the $\pi^{0} \pi^{0}$-invariant mass spectrum obtained in the reaction $p n \rightarrow d \pi^{0} \pi^{0}$ at $\sqrt{s}=2.38 \mathrm{GeV}$ [6]. Relative to a pure phase-space distribution, the data exhibit a very pronounced low-mass enhancement - the $\mathrm{ABC}$ effect.

Moreover, the new measurements discovered a strict correlation between the appearance of the $\mathrm{ABC}$ effect and the appearance of the $d^{*}(2380)$ resonance structure in the total cross sections of the fusion reactions to $d$, 


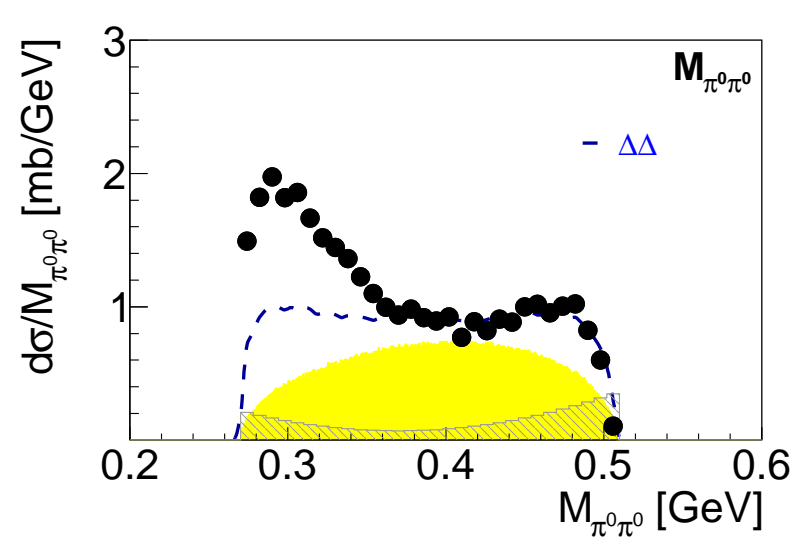

FIG. 1: (color online). $\pi^{0} \pi^{0}$ invariant mass distribution in the $p n \rightarrow d \pi^{0} \pi^{0}$ reaction at $\sqrt{s}=2.38 \mathrm{GeV}$, the $d^{*}(2380)$ resonance peak energy region. Black solid circles represent the data and the hatched area an estimate of systematic uncertainties (from Ref. [6]). The yellow shaded area denotes pure phase space and the dashed line gives a calculation of the conventional $t$-channel $\Delta \Delta$ excitation process $p n \rightarrow \Delta \Delta \rightarrow d \pi^{0} \pi^{0}$, both normalized arbitrarily in height.

${ }^{3} \mathrm{He}$ and ${ }^{4} \mathrm{He}$, if these reactions were associated with the production of an isoscalar pion pair [5, 7, 12, 14].

In contrast to these findings no $\mathrm{ABC}$ effect was observed in the non-fusion reactions $p n \rightarrow p p \pi^{0} \pi^{-}$and $p n \rightarrow p n \pi^{0} \pi^{0}$ despite of the appearance of $d^{*}(2380)$ in these reactions. For the first reaction the non-appearance of the ABC effect is easily understood, since in this case the pions of the isovector pion pair must be in relative $p$-wave suppressing thus any low-mass enhancement. For the second reaction there is no such obvious reason.

In the following we examine possible scenarios as suitable explanations of the ABC effect. To this end we confront several phenomenological ansatzes with the observations made - with special emphasis on the $n p \rightarrow d \pi^{0} \pi^{0}$ and $n p \rightarrow n p \pi^{0} \pi^{0}$ reactions, where the largest tensions for a common explanation exist. Hence the work presented in the following is intended to be primarily a phenomenological search for a reasonable solution rather than presenting here already a fundamental theoretical treatment of this issue, which would need to be a next step as soon as a reasonable phenomenological solution has been found.

The aim of this paper is also to document calculations of the $p n \rightarrow d^{*} \rightarrow \Delta \Delta$ route, the results of which were shown in previous publications [6, $[],,[9,10]$.

\section{HYPOTHESES FOR ITS EXPLANATION}

From the Dalitz plots of the double-pionic fusion reactions [5 7 7, 12, 14] we know that $d^{*}(2380)$ decays predominantly via the $\Delta \Delta$ system in the intermediate state

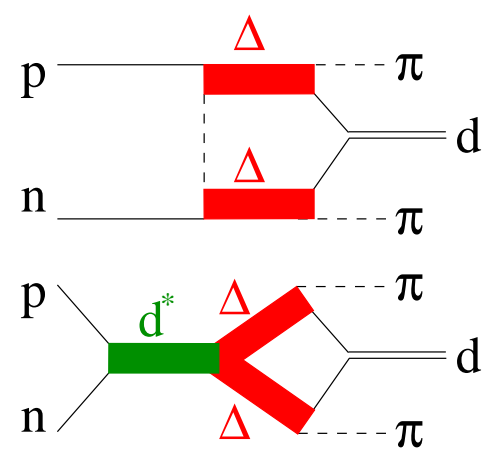

(a)

FIG. 2: (color online). Schematic representation of the processes $p n \rightarrow d \pi \pi$ via $t$-channel meson exchange (a) and $p n \rightarrow d^{*}(2380) \rightarrow \Delta \Delta \rightarrow d \pi \pi(\mathrm{b})$.

- with the two $\Delta$ s being in relative $s$-wave. This is in accordance with meanwhile numerous theoretical work about this resonance 29 39]. It also is in agreement with the measured branchings of the $d^{*}$ decay into the diverse $N N \pi \pi$ channels [40].

Therefore it seems likely that the appearance of the ABC effect is correlated with the appearance of the $\Delta \Delta$ system in the intermediate state and that way also with the internal structure of $d^{*}(2380)$.

One of the first attempts to explain the $\mathrm{ABC}$ effect was its connection with the conventional $t$-channel $\Delta \Delta$ excitation, a schematic diagram of which is depicted in Fig. 2(a). As pointed out already by Risser and Shuster [41], such an excitation causes a double-hump structure of the $\pi \pi$-invariant mass spectrum with enhancements both at low and high masses relative to phase space. If the center-of-mass energy $\sqrt{s}$ is below the mass $2 m_{\Delta}$, then the low-mass enhancement tends to be more prominent - see Fig. 3. If it is above $2 m_{\Delta}$, then the high-mass enhancement dominates.

Fig. 10xhibits the $\pi^{0} \pi^{0}$-invariant mass $\left(M_{\pi^{0} \pi^{0}}\right)$ spectrum at resonance in the $p n \rightarrow d \pi^{0} \pi^{0}$ reaction, where the background from conventional processes is small compared to the resonance signal and can be neglected in the following. The dashed curve shows the result, if we calculate the $t$-channel $\Delta \Delta$ process (Fig. $2(\mathrm{a})$ ) as given by Risser and Shuster [41].

For comparison we show in Fig. [3] data [6] for the $M_{\pi^{0} \pi^{0}}$ spectrum at $\sqrt{s}=2.5 \mathrm{GeV}$, i.e., at an energy above the $d^{*}(2380)$ region in the regime of the conventional $t$-channel $\Delta \Delta$ process. The data are accounted reasonably well by the calculations in the framework of Risser and Shuster. Since $\sqrt{s}>2 m_{\Delta}$ here, the high-mass enhancement is now dominating and correctly reproduced by these calculations. To the observed low-mass enhancement also the high-energy tail of $d^{*}(2380)$ may contribute - as well as possibly a $\Delta \Delta$ final-state interaction as discussed below. 


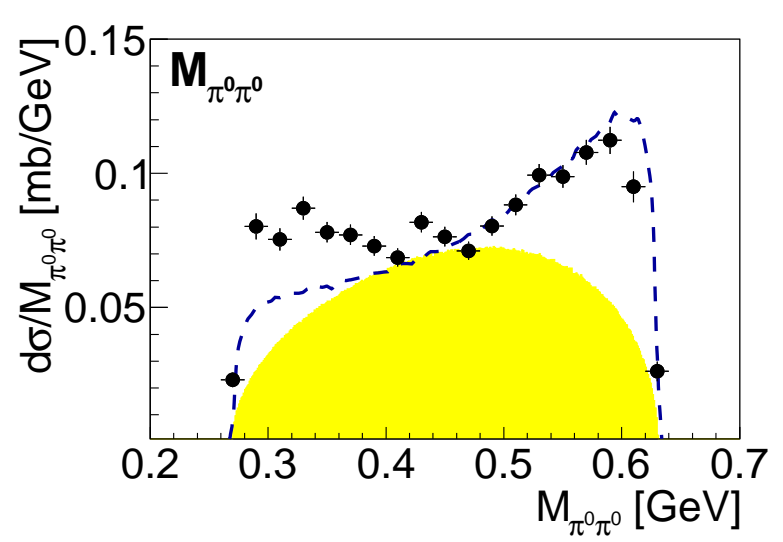

FIG. 3: (color online). The same as Fig. 1, but at $\sqrt{s}=$ $2.5 \mathrm{GeV}$, i.e., above the $d^{*}(2380)$ region in the regime of the $t$-channel $\Delta \Delta$ process. The data are from Ref. [6].

\section{Description of the $d^{*}(2380)$ Resonance Process}

In the following we give the formalism for calculating the $s$-channel resonance process

$$
p n \rightarrow d^{*}(2380) \rightarrow \Delta \Delta \rightarrow d \pi^{0} \pi^{0}
$$

depicted schematically in Fig. 2(b) and as used for the calculations presented in Ref. [6, 7, 9, 10].

The differential cross section is then given by

$$
d \sigma=\frac{(2 \pi)^{4}}{4 p_{i} \sqrt{s}} \sum\left|A\left(m_{p}, m_{n}, m_{d}, \hat{k}_{1}, \hat{k}_{2}\right)\right|^{2} d \Phi_{3},
$$

where $d \Phi_{3}$ denotes the 3-body phase-space element and the sum runs over the observable spin substates $m_{p}, m_{n}, m_{d}$ of proton, neutron and deuteron, as well as over all kinematical variables not considered explicitly in the dependence of the differential cross section $d \sigma . \vec{k}_{1}$ and $\vec{k}_{2}$ denote the momentum vectors of the emitted pions and $p_{i}$ is the momentum in the initial $p n$ channel.

The transition amplitude $A\left(m_{p}, m_{n}, m_{d}, \hat{k}_{1}, \hat{k}_{2}\right)$ is given by

$$
A\left(m_{p}, m_{n}, m_{d}, \hat{k}_{1}, \hat{k}_{2}\right) \sim \int M_{R} * \hat{\varphi}_{d} d \vec{q},
$$

where $q$ denotes the relative momentum between the two nucleons merging finally into the deuteron and $\hat{\varphi}_{d}$ denotes the deuteron wave function in momentum space. Its square provides the probability distribution for finding the two involved nucleons with momenta $p_{N_{1}}$ and $p_{N_{2}}$. In our numerical calculations we used $q_{\max }=800$ $\mathrm{MeV} / \mathrm{c}$ as an upper limit for the relative momentum $q$. Extending the upper limit to $1.5 \mathrm{GeV} / \mathrm{c}$ yields no significant changes in the calculated observables, which would be of relevance for the considerations in the following. Since we are interested here only in the shape of the $\pi \pi$ invariant mass distribution, we omit constants and use the proportionality sign in eq. (3).

The matrix element $M_{R}$ is given by

$$
M_{R}\left(m_{p}, m_{n}, m_{d}, \hat{k}_{1}, \hat{k}_{2}\right)=M_{R}^{0} \Theta_{R}\left(m_{p}, m_{n}, m_{d}, \hat{k}_{1}, \hat{k}_{2}\right),
$$

where the function $\Theta$ contains the substate and angular dependent part, and

$$
M_{R}^{0}=D_{R} * D_{\Delta_{1}} * D_{\Delta_{2}}
$$

Here $D_{\Delta}$ denotes the $\Delta$ propagator

$$
D_{\Delta}=\frac{\sqrt{m_{\Delta} \Gamma_{\Delta} / q_{\pi}^{N \pi}}}{M_{N \pi}^{2}-m_{\Delta}^{2}+i m_{\Delta} \Gamma_{\Delta}}
$$

and

$$
\Gamma_{\Delta}\left(q_{\pi}^{N \pi}\right)=\gamma\left(q_{\pi}^{N \pi}\right)^{3} \frac{R^{2}}{1+R^{2}\left(q_{\pi}^{N \pi}\right)^{2}}
$$

with $\gamma=0.74, R=6.3(\mathrm{GeV} / \mathrm{c})^{-1}$ and $q_{\pi}^{N \pi}$ denoting the decay momentum of the pion in the $\Delta$ system [41]. $M_{N \pi}$ refers to the invariant mass of the $N \pi$ system.

If we write $D_{R}$ in form of a Breit-Wigner amplitude, then we have

$$
M_{R}^{0}=\frac{m_{R} \sqrt{f \Gamma_{i} \Gamma_{\Delta \Delta}}}{s-m_{R}^{2}+i m_{R} \Gamma_{R}} D_{\Delta_{1}} D_{\Delta_{2}}
$$

with $\operatorname{mass} m_{R} \approx 2.38 \mathrm{GeV}$ and width $\Gamma_{R}\left(s=m_{R}^{2}\right) \approx$ $70 \mathrm{MeV}$.

The factor

$$
f=\frac{s}{p_{i} p_{f}} \frac{2 J+1}{\left(2 s_{p}+1\right)\left(2 s_{n}+1\right)}
$$

is the flux factor of the Breit-Wigner resonance. Here $p_{f}=p_{\Delta \Delta}$, given in eq.(11), is the decay momentum of each $\Delta$ in the $d^{*}$ system and $s_{p}$ and $s_{n}$ denote the nucleon spins in the initial channel.

$\Gamma_{i}$ and $\Gamma_{\Delta \Delta}$ denote the partial widths for formation and decay of the $s$-channel resonance. They are momentum dependent quantities and proportional to the square of the respective (effective) coupling constants $g_{p n}$ and $g_{\Delta \Delta}$.

In case of $d^{*}$ with $I\left(J^{P}\right)=0\left(3^{+}\right)$the resonance is created by the ${ }^{3} D_{3}-{ }^{3} G_{3}$ coupled partial waves between $p$ and $n$ in the initial system [1-3]. Hence we have $\Gamma_{i}=g_{p n}^{2} p_{i}^{2 L+1}$, where $L$ denotes the orbital angular momentum in the initial pn channel. Since the resonance is far beyond the $p n$ threshold, the momentum dependence over the comparatively narrow region of the resonance is small and can actually be neglected here.

In the exit channel the $d^{*}$ resonance decays into the $\Delta \Delta$ system with a relative $S$-wave between the two $\Delta \mathrm{s}$ 
- as observed in the $\Delta$ angular distribution (Fig. 5 in Ref. [6]). Therefore we have

$$
\Gamma_{\Delta \Delta}=g_{\Delta \Delta}^{2} p_{\Delta \Delta},
$$

with

$$
\begin{aligned}
2 \vec{p}_{\Delta \Delta} & =\vec{p}_{\Delta_{1}}-\vec{p}_{\Delta_{2}} \\
& =\left(\vec{p}_{N_{1}}+\vec{k}_{1}\right)-\left(\vec{p}_{N_{2}}+\vec{k}_{2}\right) .
\end{aligned}
$$

Note that the $p_{\Delta \Delta}$ dependences in eqs. (9) and (10) cancel each other, when inserted into eq. (8).

The momentum dependent total width of the resonance is then given by

$$
\begin{aligned}
& \Gamma_{R}(s)=\Gamma_{i}+\sum \Gamma_{f}=\Gamma_{i}+\gamma_{R} * \\
& \int d m_{1}^{2} d m_{2}^{2} p_{\Delta \Delta}\left|D_{\Delta_{1}}\left(m_{1}^{2}\right) D_{\Delta_{2}}\left(m_{2}^{2}\right)\right|^{2} \theta\left(s-\left(m_{1}+m_{2}\right)^{2}\right),
\end{aligned}
$$

where $m_{1}^{2}$ and $m_{2}^{2}$ denote the invariant mass-squared of the $N \pi$ pairs forming the systems $\Delta_{1}$ and $\Delta_{2}$, respectively [42]. The integral runs then over squared invariant masses from the pion emission threshold, given by $m_{i}^{2}=\left(m_{N}+m_{\pi}\right)^{2}$ in the $N N \pi \pi$ channels, to upper bounds defined by the $\theta$ function, which ensures energy conservation.

The second term in eq. (12) denotes the decays of the resonance via the intermediate $\Delta \Delta$ system. The quantity $\gamma_{R}$ contains the coupling constant $g_{\Delta \Delta}$ and other constants and is fitted to yield a total width of $\Gamma_{R}\left(s=m_{R}^{2}\right)$ $=70 \mathrm{MeV}$. In the following sections we discuss also proposed scenarios, where the intermediate state is not only $\Delta \Delta$. In such cases eq. (12) has to be complemented accordingly.

The decay of $d^{*}(2380)$ into its various $N N \pi \pi$ channels and into the $n p$ channel have been discussed in detail in Ref. [40]. There also the experimentally extracted partial widths $\Gamma_{i}\left(s=m_{R}^{2}\right)$ and $\Gamma_{f}\left(s=m_{R}^{2}\right)$ are given and can be deduced, respectively, from the quoted partial cross sections at resonance.

The angular dependence is obtained from the angular momentum coupling in entrance and exit channels:

$$
\vec{s}_{p}+\vec{s}_{n}+\vec{L}=\vec{J}=\vec{s}_{\Delta_{1}}+\vec{s}_{\Delta_{2}}
$$

with

$$
\begin{aligned}
\vec{s}_{p}+\vec{s}_{n} & =\vec{s} \\
\vec{s}_{\Delta_{1}} & =\vec{l}_{1}+\vec{s}_{N_{1}}, \\
\vec{s}_{\Delta_{2}} & =\vec{l}_{2}+\vec{s}_{N_{2}}, \\
\vec{s}_{N_{1}}+\vec{s}_{N_{2}} & =\vec{s}_{d} .
\end{aligned}
$$

Here $\vec{s}_{p}, \vec{s}_{n}$ and $\vec{s}_{d}$ with $s_{p}=s_{n}=\frac{1}{2}$ and $s_{d}=1$ denote the spins of $p, n$ and $d$. The orbital angular momenta $\vec{l}_{1}$ and $\vec{l}_{2}$ with $l_{1}=l_{2}=1$ stand for the pion $p$-waves originating from $\Delta$ decay and $\vec{L}$ is the initial orbital angular momentum between the incident proton and neutron. Since we deal here with a resonance in the ${ }^{3} D_{3}{ }^{3} G_{3}$ coupled partial waves the spin of the nucleon pair in the initial channel is $s=1$.

Eq. (13) assumes that we have $s$-wave between the two $\Delta \mathrm{s}$ in the intermediate state - in agreement with observation, as mentioned already above.

If the coordinate system is chosen to be the standard one with the z-axis pointing in beam direction (implying $m_{L}=0$ and $\left.\left(\Theta_{i}, \Phi_{i}\right)=(0,0)\right)$, then the function $\Theta_{R}\left(m_{p}, m_{n}, m_{d}, \hat{k}_{1}, \hat{k}_{2}\right)$ defined in eq. (3) is built up by the corresponding vector coupling coefficients and spherical harmonics representing the angular dependence due to the orbital angular momenta involved in the reaction:

$$
\begin{aligned}
\Theta_{R}\left(m_{p}, m_{n}, m_{d}, \quad \hat{k}_{1}, \hat{k}_{2}\right)= & \\
& \sum\left(\frac{1}{2} \frac{1}{2} m_{p} m_{n} \mid 1 m_{s}\right) \quad\left(1 L m_{s} 0 \mid J M\right) \\
& \left(J M \mid \frac{3}{2} \frac{3}{2} m_{1}^{\Delta} m_{2}^{\Delta}\right) \quad\left(\frac{3}{2} m_{1}^{\Delta} \mid \frac{1}{2} 1 m_{1}^{N} m_{1}\right) \\
& \left(\frac{3}{2} m_{2}^{\Delta} \mid \frac{1}{2} 1 m_{2}^{N} m_{2}\right) \quad\left(1 m_{d} \mid \frac{1}{2} \frac{1}{2} m_{1}^{N} m_{2}^{N}\right) \\
& Y_{L 0}(0,0) \quad Y_{1 m_{1}}\left(\hat{k_{1}}\right) \quad Y_{1 m_{2}}\left(\hat{k_{2}}\right) .
\end{aligned}
$$

The angular distributions for deuteron and pions resulting from eq. (15) are displayed in Fig. 5 of Ref.[6].

The $p$-wave pions emerging from the intermediate $\Delta \Delta$ system can couple to relative $s$ - and $d$-waves. In the first instance, $d$ and $\pi \pi$ systems must then be in relative $d$ wave to match the requirement for the resonance spin, whereas in the second case $d$ and $\pi \pi$ systems have to be in relative $s$-wave.

The calculation of the observables resulting from the process in Fig. 2(b) has been realized by use of the MonteCarlo technique. There, after generation of the deuteron momentum within the reaction phase space the momenta of the nucleons inside the deuteron are diced to be distributed according to the deuteron wave function. By subsequent use of the formulae given in eqs. $(2-15)$ the calculation then conforms to the process represented by the diagram in Fig. 2(b). The boson symmetrization has been realized by proper reshuffling of the Monte-Carlo events. [76]. The correctness of the Monte-Carlo results has been cross-checked by respective analytic calculations of the reaction amplitude corresponding to the diagram in Fig. 2(b) [42].

For the $\pi^{0} \pi^{0}$-invariant mass spectrum the calculation of this resonance process is shown by the dashed line in Fig. 4. It gives the proper tendency. However, the produced low-mass enhancement is much too small in comparison to that observed in the data.

In fact, the calculation for the $M_{\pi^{0} \pi^{0}}$ distribution is practically identical to that of the $t$-channel process depicted in Fig. 2(a) and shown by the dashed curve in Fig. 1. This is not surprising, since at the energy of in- 


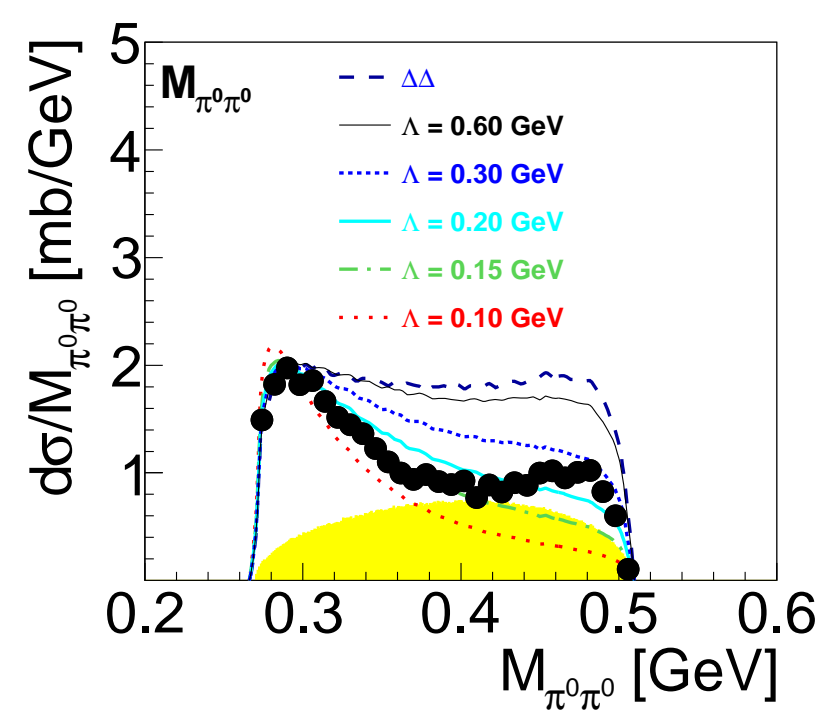

FIG. 4: (color online). Illustration of the vertex function effect on the $\pi^{0} \pi^{0}$ invariant mass distribution of the $p n \rightarrow$ $d \pi^{0} \pi^{0}$ reaction at $\sqrt{s}=2.38 \mathrm{GeV}$. Black solid circles give the data from Ref. [6], the yellow shaded area denotes pure phase space (arbitrarily normalized). The dashed line represents the calculation of the process $p n \rightarrow d^{*}(2380) \rightarrow \Delta \Delta \rightarrow d \pi^{0} \pi^{0}$ without the vertex function from eq. (16). The other lines give the calculations with vertex function for cutoff parameters $\Lambda=0.1,0.15,0.2,0.3$ and $0.6 \mathrm{GeV} / \mathrm{c}$. The curves have been normalized to the data point at $M_{\pi^{0} \pi^{0}}=0.29 \mathrm{GeV}$.

terest we are much below the nominal $\Delta \Delta$ threshold of $2 m_{\Delta}$, so that also in this case the intermediate $\Delta \Delta$ system is strongly favored to be in relative $s$-wave. Hence the dynamics of the $\pi^{0} \pi^{0}$ system observed in the $M_{\pi^{0}} \pi^{0}$ spectrum appears to be similar. And since the $t$-channel $\Delta \Delta$ process is the dominant background for the $d^{*}$ formation process, any interference between both processes will not affect the shape of the $M_{\pi^{0} \pi^{0}}$ spectrum.

\section{$d^{*} \Delta \Delta$ Vertex Function}

In order to heal the deficiency in the calculated lowmass enhancement, a vertex function at the $d^{*} \Delta \Delta$ vertex was introduced in Ref. [6] to account for finite size effects. It multiplies the expression in eq. (10) and was chosen to be of monopole type

$$
F F \sim \frac{\Lambda^{2}}{\Lambda^{2}+p_{\Delta \Delta}^{2}} .
$$

If we neglect the Fermi motion of the nucleons within the deuteron, then the nucleon momenta cancel in eq. (11) and we have $p_{\Delta \Delta}=p_{\pi^{0} \pi^{0}}$, where $p_{\pi^{0} \pi^{0}}$ is the relative pion momentum. This means that the $p_{\Delta \Delta}$ dependence of the $d^{*}$ resonance term in eq. (8) is directly reflected in the $M_{\pi^{0} \pi^{0}}$ spectrum. And since the $p_{\Delta \Delta}$ dependence in eq. (8) solely rests in the vertex function, we have here the unique situation to observe directly the vertex function in the $M_{\pi^{0} \pi^{0}}$ spectrum. In other words, the $\mathrm{ABC}$ effect in this context is nothing else but a consequence of the $d^{*} \rightarrow \Delta \Delta$ vertex function causing the observed low-mass enhancement by suppression of the high-mass region.

We note in passing that already in the work of Kälbermann and Eisenberg [43] about the ABC effect formfactors had been introduced, but there at the $\pi N N$ vertices.

In Ref. [6] the cutoff parameter $\Lambda$ had been fitted to the data in the $M_{\pi^{0} \pi^{0}}^{2}$ spectrum (Dalitz plot projection) resulting in $\Lambda \approx 0.16 \mathrm{GeV} / \mathrm{c}$, which corresponds to a length scale of $r=\frac{\hbar \sqrt{6}}{\Lambda} \approx 2 \mathrm{fm}$. Naively this could be associated with a hadronic size of $d^{*}$. However, with a binding of $80 \mathrm{MeV}$ the size of a tightly bound $\Delta \Delta$ system is expected to be considerably smaller. A possible solution of this paradox is that this tightly bound $\Delta \Delta$ state resonates with respect to the $D_{12} \pi$ configuration, which has a much lower threshold [44]. The $D_{12} \pi$ scenario will be discussed further below.

Fig. 4 shows the effect of the vertex function on the $M_{\pi^{0} \pi^{0}}$ spectrum for cutoff parameters $\Lambda=0.1,0.15,0.2$, 0.3 and $0.6 \mathrm{GeV} / \mathrm{c}$. As we see, values in the range 0.15 $0.20 \mathrm{GeV} / \mathrm{c}$ are preferred by the data.

Though this ansatz accounts very well for the data on the double-pionic fusion reactions to the deuteron (and also to ${ }^{3} \mathrm{He}$ and ${ }^{4} \mathrm{He}$ [12 15], which are not considered here), there are two disturbing features at a first glance:

First of all, the cutoff parameter $\Lambda$ appears to be unusually small. Cutoff parameters used in the description of hadronic reactions are usually three to four times larger. However, these are conventionally employed for $t$-channel exchanges, see e.g. Refs. [45, 46]. In our case of an $s$-channel resonance decay the assumed vertex function is actually identical in structure to that appearing in eq. (6) for the $\Delta \rightarrow N \pi$ decay. In fact, there we have $R^{-1}=0.16 \mathrm{GeV} / \mathrm{c}$, which is just the value of $\Lambda$ found here for the dibaryon resonance. Indeed, such vertex functions are commonly used for the description of the decay of baryon resonances, see e.g. Ref. [47, 48], with cutoff parameters being similarly small as the value obtained here.

Second, for non-fusion reactions like $p n \rightarrow p n \pi^{0} \pi^{0}$ the vertex function in eq. (16) does not affect primarily the $M_{\pi^{0} \pi^{0}}$ spectrum, but the $M_{p n}$ spectrum, as discussed in Ref. [10], since the unbound pn system is no longer restricted in its relative motion by the deuteron wave function. The fact that the vertex function does not influence significantly the $M_{\pi^{0} \pi^{0}}$ spectrum actually agrees perfectly with the experimental finding that there is no significant $\mathrm{ABC}$ effect in this reaction [10].

However, the impact of the vertex function on the $M_{p n}$ spectrum has been demonstrated in Ref. [10] to exaggerate the enhancement of the low-mass region in the $M_{p n}$ 
spectrum tending thus to be at variance with the data [10] - see Fig. 7 in Ref. 10.

In this work the resonance contribution to the $n p \rightarrow$ $n p \pi^{0} \pi^{0}$ reaction was calculated in analogy to that for the $n p \rightarrow d \pi^{0} \pi^{0}$ reaction, only that three-body phasespace is replaced by a four-body phase space and the condition for the momenta of the emitted nucleons to be in accord with the deuteron wave function is replaced by the requirement that the emerging $n p$ pair undergoes a final-state interaction chosen to be of Migdal-Watson type [49 51]:

$$
F S I=1+\frac{R^{-2}}{\left(-\frac{1}{a_{s}}+\frac{1}{2} r_{0} p_{n p}^{2}\right)^{2}+p_{n p}^{2}},
$$

which multiplies the expression for the cross section. Here $\mathrm{R}$ denotes the vertex size, $r_{0}$ the effective range and $a_{s}$ the isoscalar triplet scattering length. For these the values $2.75 \mathrm{fm}, 1.75 \mathrm{fm}$ and $+5.4 \mathrm{fm}$ have been used. Whereas the latter two conform to the experimentally known values for effective range and scattering length, the value of $\mathrm{R}$ has been taken to be identical to the one used in $p p$ induced two-pion production [52].

Re-inspecting that calculation [10] in the course of this work we have noted that by mistake the vertex function was applied twice there. The updated calculation is plotted in Fig. 5 by the solid curve. The dotted curve gives the calculation without FSI and without vertex function, the dashed curve shows the calculation with FSI, but without vertex function. We still have the situation that the calculation without vertex function appears to be slightly superior to that with vertex function. However, accounting for the substantial systematic uncertainties in the experimental $M_{p n}$ spectrum (hatched areas in Fig. (5) the calculation with vertex function is appropriate as well. We also note that for the FSI in these calculations it has been assumed that all emitted $p n$ pairs are in relative $s$-waves, which is supported by the observation, that the $M_{p n}$ spectrum cumulates at small invariant masses.

We just note in passing that - different from the $n p \rightarrow d \pi^{0} \pi^{0}$ reaction - the $n p \rightarrow n p \pi^{0} \pi^{0}$ reaction contains also isovector contributions from $t$-channel background processes. In principle one might ask, whether they could possibly be the cause of the different behavior of the $M_{\pi^{0} \pi^{0}}$ spectra in both reactions. In the $d^{*}$ resonance region the background processes make up about thirty percent of the total cross section. Isospin decomposition according to Refs. [53 55] gives roughly half of the background processes to be isovector, i.e. about $15 \%$ of the total cross section. Since isoscalar and isovector contributions do not interfere in total cross section and invariant-mass spectra, the influence of the isovector part there is small. Moreover, $d^{*}$ and $t$-channel background processes give nearly identical shapes for the $M_{\pi^{0} \pi^{0}}$ spectrum - see Fig. 7 in Ref. [10].
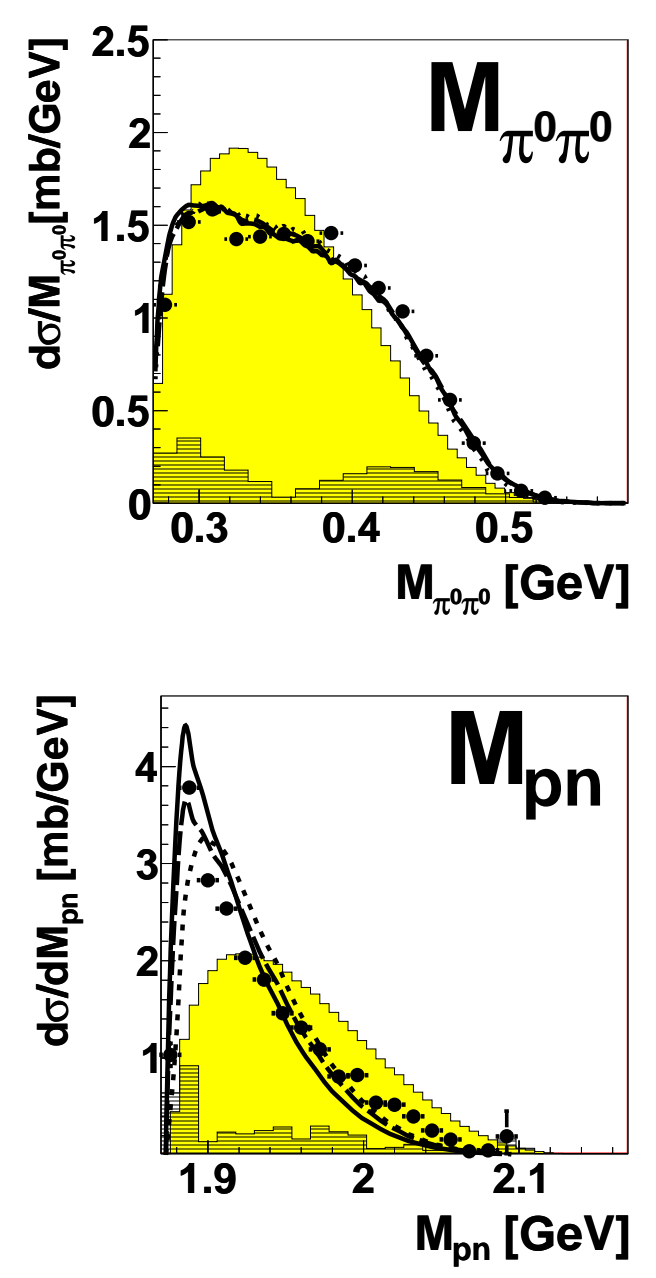

FIG. 5: (color online). Differential distributions of invariant masses $M_{\pi^{0} \pi^{0}}$ (top) and $M_{p n}$ (bottom) for the $n p \rightarrow n p \pi^{0} \pi^{0}$ reaction. The black solid circles denote data [10], the shaded areas indicate the estimate of systematic uncertainties and the yellow shaded areas phase-space distributions. The solid (dashed) lines give the calculation of the reaction process including the route $n p \rightarrow d^{*}(2380) \rightarrow \Delta \Delta \rightarrow n p \pi^{0} \pi^{0}$ with (without) use of the vertex function in eq. (16). The dotted line is a calculation without both vertex function and FSI. Note that in the $M_{\pi^{0} \pi^{0}}$ spectrum all lines lie nearly on top of each other.

In the following we examine three alternative ansatzes, which have been given recently in the literature for the explanation of the $\mathrm{ABC}$ effect. We also discuss as a new aspect the possibility of a $D$-state between the $\Delta \mathrm{s}$ in the intermediate $\Delta \Delta$ system.

We do not deal here with the model of Gardestig, Fäldt and Wilkin [57], since this has been designed specifically for the $\mathrm{ABC}$ effect in the double-pionic fusion to ${ }^{4} \mathrm{He}$ and is not applicable to the basic double-pionic fusion to the deuteron. 


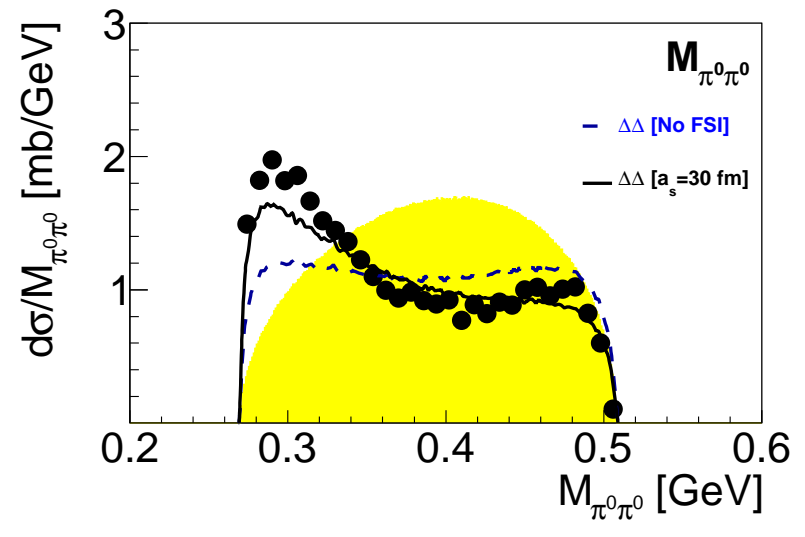

FIG. 6: (color online). The same as Fig. 4 but the lines represent now the effect of the $\Delta \Delta$-FSI in dependence of the scattering length.

\section{$\Delta \Delta$ Final State Interaction}

Another, alternative attempt to explain the ABCeffect by a final-state interaction (FSI) between the two $\Delta \mathrm{s}$ in the intermediate state dates back to the first WASA measurements on this issue [13, 56]. We note that this ansatz dates back to the time, when it was not yet known that the $\mathrm{ABC}$ effect is correlated with the presence of the $s$-channel resonance $d^{*}(2380)$. Though including a FSI for ejectiles, which beforehand formed a resonance, has the threaten of double-counting, we will discuss this scenario for historical reasons - and also because its effect is formally very similar to that of the vertex function.

In Refs. [13, 56] the $\Delta \Delta$-FSI was parameterized in the Migdal-Watson ansatz [49 51] providing the factor analogous to eq. (17) -

$$
F S I=1+\frac{R^{-2}}{\left(-\frac{1}{a_{s}}+\frac{1}{2} r_{0} p_{\Delta \Delta}^{2}\right)^{2}+p_{\Delta \Delta}^{2}},
$$

which multiplies the expression for the cross section. For decent values of $r_{0}=2 \mathrm{fm}$ and $R=1.1 \mathrm{fm}$ a reasonable description of the $M_{\pi^{0} \pi^{0}}^{2}$ spectrum is achieved with $a_{s} \approx 30 \mathrm{fm}$, see Fig. 6] Numerically the first term in eq. (18) is small compared to the second one and also the finite range term in the denominator of the second term may be neglected. Thus we see that eqs. (16) and (18) both have a similar structure with similar $p_{\Delta \Delta}$ dependence, i.e. they lead to practically the same corrections in the differential spectra.

Thus eq. (18) formally leads back to eq. (16) for the vertex function. Actually, this ansatz seems to be independent of the existence of the $d^{*}$ resonance and hence also valid for $t$-channel $\Delta \Delta$ excitations, where no ABC enhancements are observed. However, the scattering length in eq. (18) depends on the involved partial wave with isospin I and total spin J. The fact that the deuteron angular distribution gets flatter again above the $d^{*}$ region [58] in the $t$-channel $\Delta \Delta$ regime means that there are different partial waves involved, which necessarily do not need to have a large scattering length.

$$
d^{*}(2380) \rightarrow d \sigma \text { decay }
$$

Recently Platonova and Kukulin proposed an alternative explanation of the ABC effect [59,60]. They consider two possible decay branches for the $d^{*}(2380)$ resonance. Hereby they go back to the work of Dyson and Xuong [29], who - based on SU(6) - predicted a sextet of nonstrange dibaryon states. In that work $d^{*}(2380)$ is denoted by $D_{03}$, where the first index means the isospin and the second one the spin of the dibaryon state.

Kukulin and Platonova assume the main decay of $D_{03}$ to proceed via the $D_{12}$ member of the sextet, i.e. $D_{03} \rightarrow D_{12} \pi \rightarrow d \pi \pi$, where $D_{12}$ is identified with the $I\left(J^{P}\right)=1\left(2^{+}\right)$state at the $N \Delta$ threshold, which produces a pole in the ${ }^{1} D_{2}$ partial wave of $p p$ scattering. For the numerous discussions, whether this state constitutes a true $s$-channel resonance or not, see e.g. Refs. [61 72]. Since in this route $D_{12}$ and the associated pion have to be in relative $p$-wave, in order to fulfill the angular momentum requirements of $D_{03}$, this decay route is practically indistinguishable from the route $D_{03} \rightarrow \Delta \Delta \rightarrow d \pi \pi$, i.e. both routes give essentially identical results for the $M_{\pi^{0} \pi^{0}}$ spectrum (dashed lines in Fig. 7).

Note that in Ref. [59 momentum dependent decay widths containing also vertex functions analogous to that in eq. (16) have been used with very small cutoff parameters $\Lambda=0.1-0.2 \mathrm{GeV} / \mathrm{c}$. Since the decays $d^{*} \rightarrow D_{12} \pi$ and $D_{12} \rightarrow d \pi$ are $p$-wave decays, which primarily go with the third power of the decay momentum, a vertex function with very small cutoff parameter tends to cancel this momentum dependence. Hence the calculation of the decay route $d^{*} \rightarrow D_{12} \pi \rightarrow d \pi \pi$ with very small cutoff parameters gets very similar to that of $d^{*} \rightarrow \Delta \Delta \rightarrow d \pi \pi$ without the use of a vertex function for the $d^{*} \Delta \Delta$ decay vertex.

The second decay route constitutes a really new piece in trying to explain the $\mathrm{ABC}$ effect. It assumes that a $\sigma$ meson is emitted according to $D_{03} \rightarrow d \sigma$. Due to $J^{P}=3^{+}$the $\sigma$ meson and the deuteron have to be in relative $d$-wave. This means that the transition amplitude of this process should be proportional to the relative momentum squared, i.e. $A \sim q_{\sigma-d}^{2} \sim\left(M_{\pi \pi^{\max }}^{2}-M_{\pi \pi}^{2}\right)$. This momentum dependence provides a high-mass $M_{\pi \pi}$ suppression (dotted line in Fig. 77(a)). In total, both routes if they are allowed to interfere constructively - produce an enhancement of the low-mass region relative to the high-mass region as required for the ABC effect (solid line in Fig. 7(a)). Only, the shape of the produced lowmass enhancement comes out too broad in comparison to the data. 

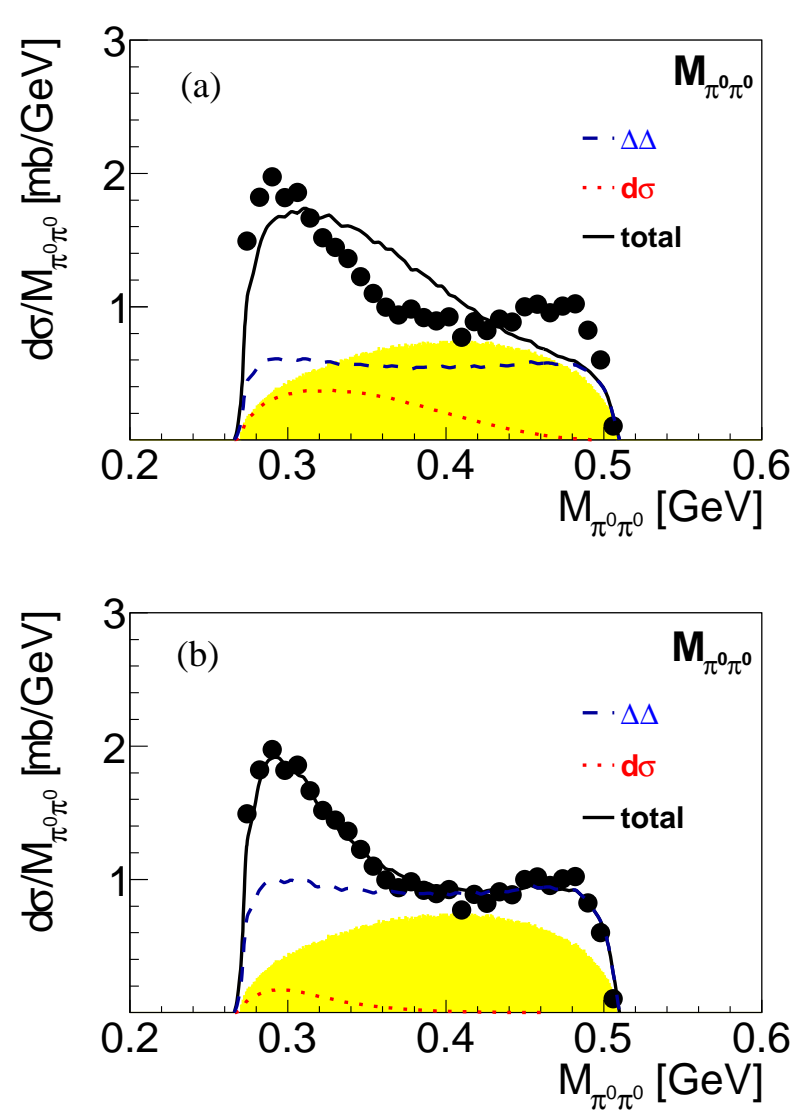

FIG. 7: (color online). The same as Fig. 4 but the lines represent now the model ansatz of Kukulin and Platonova 59] for the $d^{*}(2380)$ decay. The contribution of the main route $d^{*}(2380) \rightarrow D_{12} \pi^{0} \rightarrow d \pi^{0} \pi^{0}$ is given by the blue dashed line, which to very good approximation is represented by a $\Delta \Delta$ calculation without vertex function. The red dotted lines show the contribution of the route $d^{*}(2380) \rightarrow d \sigma \rightarrow d \pi^{0} \pi^{0}$ - in the top panel (a) for mass and width of the $\sigma$ meson according to PDG 73] $\left(m_{\sigma}=440 \mathrm{MeV}, \Gamma_{\sigma}=544 \mathrm{MeV}\right)$, and in the bottom panel (b) for the values $m_{\sigma}=300 \mathrm{MeV}$ and $\Gamma_{\sigma}=100 \mathrm{MeV}$ as used in Ref. [59]. The solid lines give the coherent sum.

In this ansatz the data are only reproduced quantitatively, if a light and narrow $\sigma$ meson $\left(M_{\sigma}=300 \mathrm{MeV}\right.$, $\Gamma_{\sigma}=100 \mathrm{MeV}$ ) is assumed (solid line in Fig. 7(b)). The strong mass reduction compared to the accepted value [73] has been assigned to chiral symmetry restoration inside the $d^{*}$ dibaryon [59].

An admixture of this decay route as small as $5 \%$ on the cross section level is enough to reproduce the ABC effect in double-pionic fusion reactions. A problem with this ansatz may arise for the $n p \rightarrow n p \pi^{0} \pi^{0}$ reaction, where the data exhibit no $\mathrm{ABC}$ effect in the $M_{\pi^{0} \pi^{0}}$ spectrum. Since in this reaction an isoscalar pion pair is produced, which can form a $\sigma$ meson, there is no obvious reason, why the $D_{03} \rightarrow$ pn $\sigma$ route should be suppressed. However, in Ref. [60] it is argued qualitatively that due to the

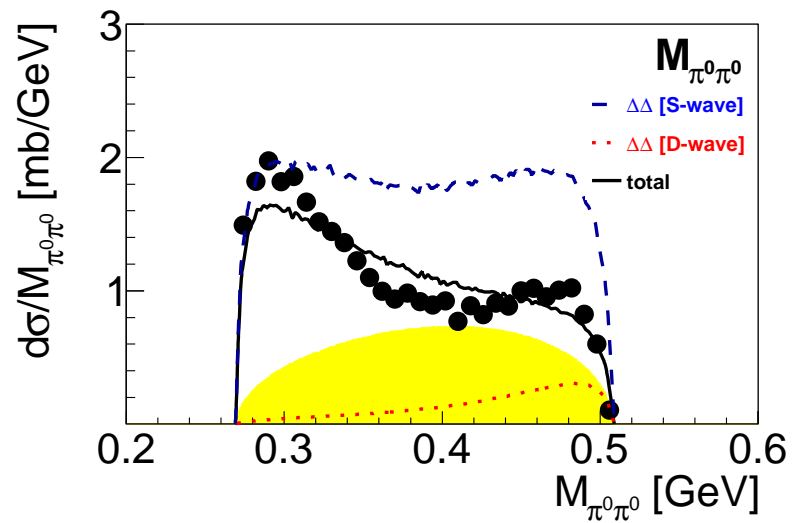

FIG. 8: (color online). The same as Fig. 4, but the dotted line represents now a $5 \%$ contribution of the $D$-wave in the intermediate $\Delta \Delta$ system and the solid curve the coherent sum of $S$ - and $D$-wave contributions - without vertex function.

much increased phase space for the unbound $N N$ system the centrifugal barrier for the $d$-wave emission of the $\sigma$ meson plays here a much bigger role and might suppress its emission more than in the $d \pi \pi$ case. Unfortunately, a convincing quantitative calculation has not yet been presented by these authors.

\section{$D$-wave $\Delta \Delta$ admixture}

Having learned in the previous example that a $D$-wave introduces a strong momentum dependence such that the $\mathrm{ABC}$ effect may be reproduced, we may consider yet another ansatz, which possibly is more realistic.

Similar to the fact that the deuteron groundstate contains a $D$-wave admixture, we may postulate also a such one for the intermediate $\Delta \Delta$ system. In principle a $D$ wave between the two $\Delta$ s may couple to spin $S=1$ and 3 states of the $\Delta \Delta$ system for a total angular momentum of $J=3$. For simplicity we consider here just the spin $S=3$ case as done in Refs. [36, 37].

The $D$-wave $\Delta \Delta$ amplitude is proportional to $q_{\Delta \Delta}^{2}$. So contrary to the ansatz with the $\Delta \Delta$ vertex function and $\Delta \Delta$ FSI, respectively, the $M_{\pi \pi}$ spectrum will be suppressed in particular at small masses, see dotted line in Fig. 8. A destructive interference between $D$-wave and $S$-wave (dashed line) components will suppress then high $M_{\pi \pi}$ invariant masses. A $20 \%$ admixture of the $D$-wave component on the amplitude level, i.e. $4-5 \%$ on the cross section level, is sufficient for a reasonable description of the ABC-effect. This means a very similar amount of $D$-wave admixture as in the deuteron groundstate.

The angular distributions are not affected noticeably by the $D$ wave $\Delta \Delta$ admixture, since the deuteron angular distribution is characterized by the total angular momentum of $d^{*}$ and the pion angular distribution by 

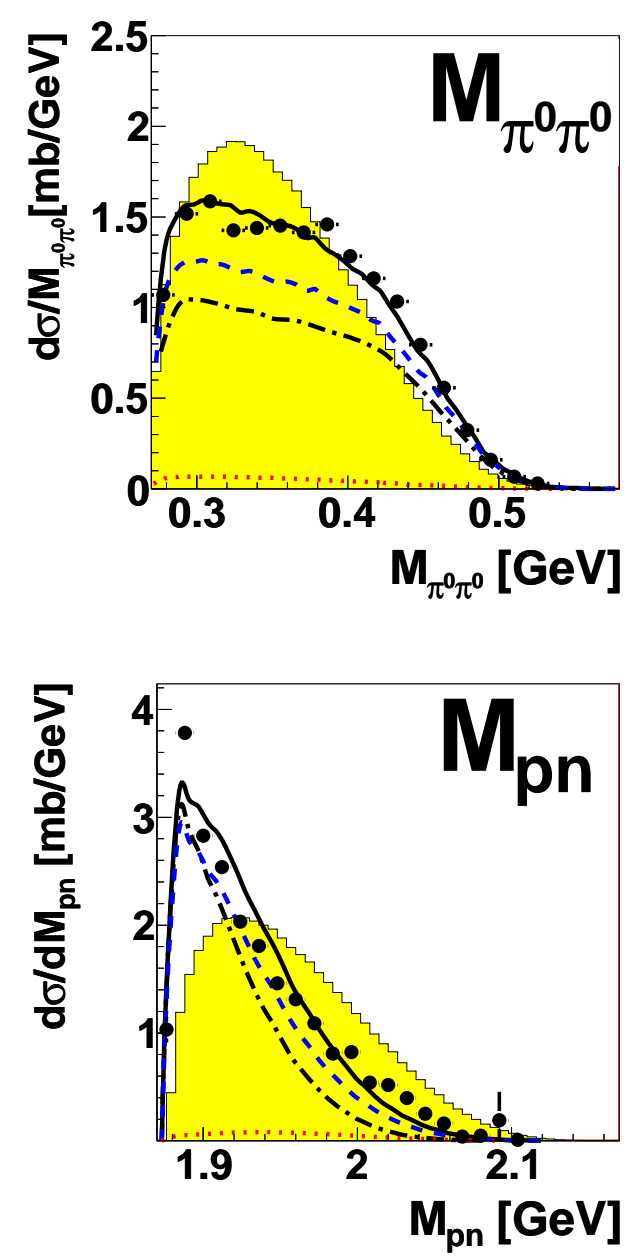

FIG. 9: (color online). The same as Fig. 5 for the $n p \rightarrow$ $n p \pi^{0} \pi^{0}$ reaction, but dashed and dotted lines represent now the $d^{*}$ contributions originating from $S$ and $D$-waves in the intermediate $\Delta \Delta$ system, respectively. The dash-dotted curves show their coherent sum. The solid lines give the full result including background contributions.

the $p$-wave decay of the intermediate $\Delta$ excitations [6].

Opposite to the previous cases, this ansatz does not cause a problem in the description of the $n p \rightarrow n p \pi^{0} \pi^{0}$ reaction. It does neither produce an $\mathrm{ABC}$ effect in the $M_{\pi^{0} \pi^{0}}$ spectrum nor an exaggerated low-mass enhancement in the $M_{p n}$ spectrum. The situation is depicted in Fig. 9, where the data from Ref. [10] are compared with the results of the ansatz with $S$ - (dashed) and $D$-wave (dotted) $\Delta \Delta$ configurations. Whereas these calculations produce practically identical shapes in the $M_{\pi^{0} \pi^{0}}$ spectrum, they differ somewhat for the $M_{p n}$ case. The solid lines give the full result, i.e. the coherent sum of $S$ and $D$-wave contribution together with the $t$-channel background processes. The data of the $n p \rightarrow n p \pi^{0} \pi^{0}$ reaction are well reproduced by this ansatz.

As discussed already in connection with the vertex

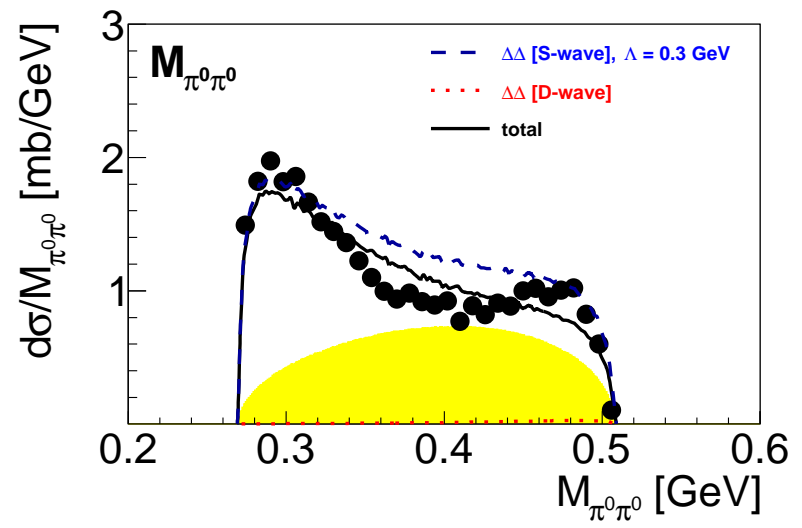

FIG. 10: (color online).The same as Fig. 8 but the dotted line represents now a only $1.5 \%$ contribution of the $D$-wave in the intermediate $\Delta \Delta$ system and the solid curve the coherent sum of $S$ - and $D$-wave contributions incorporating now also a vertex function with $\Lambda=0.3 \mathrm{GeV} / \mathrm{c}$.

function and in Ref. [9] - the $n p$ final system in the unbound case is no longer restricted by the deuteron wave function. Different from the bound nucleus case, where the relative momentum between the two $\Delta \mathrm{s}$ is essentially made up by the relative momentum between the two emerging pions, the relative $\Delta \Delta$ momentum in the unbound case is mainly transferred to the two emerging nucleons, the heavy partners of the $\Delta$ decays. Hence the introduction of $q_{\Delta \Delta}^{2}$ has no visible influence on the shape of the $M_{\pi^{0} \pi^{0}}$ spectrum, but rather on the $M_{p n}$ spectrum - see Fig. 9, bottom.

A $\Delta \Delta D$-wave contribution has actually been predicted in quark model calculations recently [36, 37]. The predicted $d$-wave percentage, however, is only $1.5 \%$. In fact, we may also reproduce the $\mathrm{ABC}$ effect with such a small $D$-wave admixture, if we incorporate also the vertex function in this ansatz and increase the cutoff parameter to $\Lambda=0.3 \mathrm{GeV} / \mathrm{c}$ - see Fig. 10. That way we may arrive at a consistent description of the $\mathrm{ABC}$ effect, which leads also to an optimal description of both $n p \rightarrow d \pi^{0} \pi^{0}$ and $n p \rightarrow n p \pi^{0} \pi^{0}$ reactions - though the interplay between cutoff parameter and $D$-wave contribution allows quite some freedom, as long as one of them is not fixed by other means.

$$
d^{*}(2380) \rightarrow N^{*}(1440) N \text { decay }
$$

This scenario has been considered to some extent in Ref. [74]. Since the $d^{*}(2380) \rightarrow N N$ decay is sizable [2] and the Roper resonance $N^{*}(1440)$ has the same quantum numbers as the nucleon, one may assume that a $d^{*}(2380) \rightarrow N^{*}(1440) N$ decay branch might also exist. Due to the spin-parity $J^{P}=3^{+}$of the $d^{*}(2380)$ resonance one needs to have at least a $D$-wave between the nucleon 


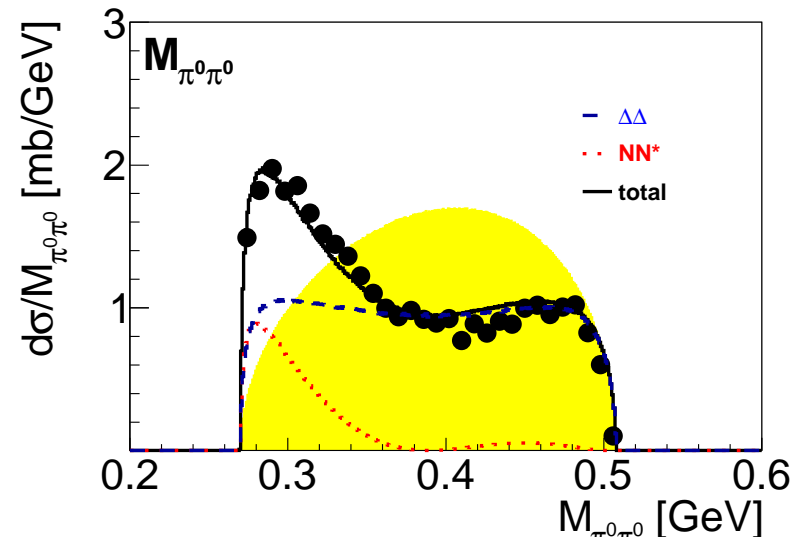

FIG. 11: (color online). Same as Fig. 4, but dashed (blue)1 and dotted (red) lines show $d^{*} \rightarrow \Delta \Delta \rightarrow d \pi \pi$ and $d^{*} \rightarrow$ $N^{*} N \rightarrow \Delta \pi N \rightarrow d \pi \pi$ contributions respectively - without applying a vertex function at the decay vertex. The solid line represents their coherent sum.

and the Roper resonance, which in the course of its decays $N^{*}(1440) \rightarrow N \sigma \rightarrow N \pi \pi$ and $N^{*}(1440) \rightarrow \Delta \pi \rightarrow N \pi \pi$ finally must transform to a $D$-wave between pion and nucleon pairs, if the nucleons fuse to a deuteron. This affords substantial reshuffling of angular momenta.

Also since the mass of $d^{*}(2380)$ is just at the $N^{*}(1440) N$ threshold, we can not expect that the amplitude for a decay into a intermediate $N^{*}(1440) N$ system in relative $D$-wave is large. But let us assume that it exists in some sizable amount. Then it will interfere with the main decay branch $d^{*}(2380) \rightarrow \Delta \Delta$. In fact, assuming a $5 \%$ decay branch via the Roper resonance the ABC-effect can be again very well reproduced - see Fig. 11.

Since in the $n p \rightarrow n p \pi^{0} \pi^{0}$ reaction the emitted $n p$ pair is no longer forced to be in relative $S$ wave, the $d^{*} \rightarrow N N^{*}$ decay even could be enhanced in this channel. The effect of this decay branch in $\pi^{0} \pi^{0}$ - and $p n$-invariant mass spectra is similar to that discussed for the $D$-wave $\Delta \Delta$ scenario.

If true that decays of baryonic excitations should have a vertex function in general, then also in this scenario the main decay route via $\Delta \Delta$ will already produce the main part of the $\mathrm{ABC}$ effect with the consequence that the necessary amount of $d^{*} \rightarrow N N^{*}$ decay is diminished to a small percentage similar to the situation discussed for the $D$-wave $\Delta \Delta$ scenario above.

If this scenario is correct then we expect also a decay of $d^{*}(2380)$ into the $N N \pi$ channel, since the decay of the Roper resonance into $N \pi$ has a branching of 55 $75 \%$ [73].

\section{CONCLUSIONS}

We have discussed several possible reasons for the $\mathrm{ABC}$ effect. Most of these hypotheses can be tuned to reproduce at least qualitatively the measured low-mass enhancements in the $M_{\pi \pi}$ spectra of double-pionic fusion reactions. The non-occurrence of the $\mathrm{ABC}$ effect in the non-fusion reaction $n p \rightarrow n p \pi^{0} \pi^{0}$ has been used as a further constraint to filter out viable solutions.

Most natural and straightforward appears the explanation of the $\mathrm{ABC}$ effect as a direct consequence of the vertex function in the $d^{*} \rightarrow \Delta \Delta$ decay vertex. In this conception the $M_{\pi \pi}$ spectrum just maps the momentum dependence of the resonance decay, which is given by that of the vertex function. The necessary cutoff parameter happens to coincide with that for the $\Delta$ resonance and is in the bulk part of values used for other baryonic excitations. This scenario also naturally explains the non-appearance of the $\mathrm{ABC}$ effect in non-fusion reactions. There remains some tension in the description of the $M_{p n}$ spectrum in the $n p \rightarrow n p \pi^{0} \pi^{0}$ reaction. However, this is still within the systematic uncertainties of the particular measurement.

Alternative hypotheses deal with postulated small $D$ wave admixtures either in the intermediate $\Delta \Delta$ system or in form of $d \sigma$ or $N^{*}(1440) N$ decay branches. A $D$-wave admixture in the intermediate $\Delta \Delta$ system has been predicted from quark-model calculations [36, 37] - though a factor of three less than needed for an exclusive explanation of the $\mathrm{ABC}$ effect without the use of the vertex function. However, if also in this scenario a vertex function is incorporated - as needed for a consistent description of baryonic excitations, then a $1.5 \% d$-wave admixture in agreement with model predictions is sufficient for a quantitative description of both key reactions.

With regard to a possible $d^{*}(2380) \rightarrow N^{*}(1440) N$ decay a dedicated experimental test of such a decay could be the investigation of isoscalar single-pion production in the energy region of interest. In addition, investigation of such a decay branch into the $N N \pi$ system can resolve the question, whether the main decay route is $d^{*}(2380) \rightarrow \Delta \Delta$ or $d^{*}(2380) \rightarrow D_{12} \pi$ as anticipated in Refs. [31, 32, [59]. Since $D_{12}$ has a sizeable decay branch into $N N$, the route via $D_{12}$ will feed also the $N N \pi$ channel, whereas such a feeding is very unlikely to happen with sizeable intensity in case of an intermediate isoscalar $\Delta \Delta$ system.

Unfortunately, there are no data at all in this energy region and even at lower energies the data base is not very precise [75]. However, though WASA at COSY has finished data taking, there are still data samples from previous runs available, which - though collected primarily for other reasons - could allow to extract the wanted isoscalar single-pion production. An analysis of such data is in progress. 
Thus the ABC effect in double-pionic fusion reactions together with its absence in non-fusion reactions may lead to some insight into the internal structure of the $d^{*}(2380)$ resonance. This is also an important aspect in the photo excitation $\gamma d \rightarrow d^{*}(2380)$. By use of virtual $\gamma \mathrm{s}$ even the transition formfactor may be obtained, which contains information about size and internal structure of this resonance state. Dedicated experiments with real $\gamma_{\mathrm{s}}$ are expected to be conducted at MAMI in near future.

\section{ACKNOWLEDGMENTS}

We acknowledge valuable discussions with V. Baru, A. Gal, V. Grishina, C. Hanhart, G. Kälbermann, V. Kukulin, E. Oset, G. J. Wagner and C. Wilkin. This work has been supported by DFG (CL 214/3-1) and STFC (ST/L00478X/1).

[1] P. Adlarson et al., Phys. Rev. Lett. 112, 202301 (2014).

[2] P. Adlarson et al., Phys. Rev. C 90, 035204 (2014).

[3] R. Workman, EPJ Web Conf. 81, 02023 (2014).

[4] R. L. Workman, W. J. Briscoe and I. I. Strakovsky, Phys. Rev. C 93, 045201 (2016).

[5] M. Bashkanov et al., Phys. Rev. Lett. 102, 052301 (2009).

[6] P. Adlarson et al., Phys. Rev. Lett. 106, 242302 (2011).

[7] P. Adlarson et al., Phys. Lett. B 721, 229 (2013).

[8] H. Kuc, PhD thesis, Univ. Paris Sud 2014; https://tel.archives-ouvertes.fr/tel-01156554.

[9] P. Adlarson et al., Phys. Rev. C 88, 055208 (2013).

[10] P. Adlarson et al., Phys. Lett. B 743, 325 (2015).

[11] G. Agakishiev et al., Phys. Lett. B 750, 184 (2015).

[12] P. Adlarson et al., Phys. Rev. C 91, 015201 (2015).

[13] M. Bashkanov et al., Phys. Lett. B 637, 223 (2006).

[14] P. Adlarson et al., Phys. Rev. C 86, 032201(R) (2012).

[15] S. Keleta et al., Nucl. Phys. A 825, 71 (2009).

[16] M. Bashkanov and H. Clement, Eur. Phys. J. 50, 107 (2014).

[17] N. E. Booth, A. Abashian, K. M. Crowe, Phys. Rev. Lett. 7, 35 (1961) ; 5, 258 (1960).

[18] I. Bar-Nir et al., Nucl. Phys. B 54, 17 (1973).

[19] A. Abdivaliev et al., Sov. J. Nucl. Phys. 29, 796(1979).

[20] R. J. Homer et al., Phys. Rev. Lett. 9, 72 (1964).

[21] J. H.Hall et al., Nucl. Phys. B 12, 573 (1969).

[22] J. Banaigs et al., Phys. Lett. B 43, 535 (1973).

[23] J. Banaigs et al., Nucl. Phys. B 67, 1 (1973).

[24] J. Banaigs et al., Nucl. Phys. B 105, 52 (1976).

[25] F. Plouin et al., Nucl. Phys. A 302, 413 (1978).

[26] F. Plouin, P. Fleury, C. Wilkin, Phys. Rev. Lett. 65, 690 (1990).

[27] R. Wurzinger et al., Phys. Lett. B 445, 423 (1999).

[28] for a review see A. Codino and F. Plouin, LNS/Ph/94-06

[29] F.J. Dyson and N.-H. Xuong, Phys. Rev. Lett. 13, 815 (1964).

[30] T. Kamae and T. Fujita, Phys. Rev. Lett 38, 471 (1977).

[31] A. Gal and H. Garcilazo, Phys. Rev. Lett. 111, 172301 (2013).
[32] A. Gal and H. Garcilazo, Nucl. Phys. A 928, 73 (2014).

[33] H. Huang, J. Ping and F. Wang, Phys. Rev. C 89, 034001 (2014) and references therein.

[34] H. Huang, J. Ping, C. Deng and F. Wang, Phys. Rev. C 90, 064003 (2015).

[35] Q. B. Li and P. N. Shen, J. Phys. G 26, 1207 (2000).

[36] F. Huang, Z. Y. Zhang, P. N. Shen and W. L. Wang, Chin. Phys. C 39, 071001 (2015).

[37] X. Q. Yuan, Z. Y. Thang, Y. W. Yu, P. N. Shen, Phys. Rev. C 60, 045203 (1999).

[38] Hua-Xing Chen et al., Phys. Rev. C 91, 025204 (2015).

[39] Y. Dong, P. Shen, F. Huang and Z. Zhang, Phys. Rev. C 91, 064002 (2015).

[40] M. Bashkanov, H. Clement and T. Skorodko, Eur. Phys. J. A 51, 87 (2015).

[41] T. Risser and M. D. Shuster, Phys. Lett. B 43, 68 (1973).

[42] V. Baru, V. Grishina and Ch. Hanhart, priv. comm.

[43] G. Kälbermann and J. M. Eisenberg, Nucl. Phys. A 426, 599 (1984).

[44] A. Gal, priv. comm. and to be published.

[45] L. Alvarez-Ruso, E. Oset and E. Hernandez, Nucl. Phys. A 633, 519 (1998).

[46] X. Cao, B.-S. Zou and H.-S. Xu, Phys. Rev. C 81, 065201 (2010).

[47] V. Dimitriev, O. Sushkov and C. Gaarde, Nucl. Phys. A 459, 503 (1986).

[48] S. Teis, W. Cassing, M. Effenberger, A. Hombach, U. Mosel and Gy. Wolf, Z. Phys. A 356, 421 (1997).

[49] A. B. Migdal, JETP 28, 1 (1955).

[50] K. W. Watson, Phys. Rev. 88, 1163 (1952).

[51] M. Schepkin, O. Zaboronsky and H. Clement, Z. Phys. A 345, 407 (1993).

[52] T. Skorodko et al., Phys. Lett. B 695, 115 (2011).

[53] L. G. Dakhno et al., Sov. J. Nucl. Phys. 37, 540 (1983).

[54] J. Bystricky et al., J. Physique 48, 1901 (1987).

[55] T. Skorodko et al., Phys. Lett. B 679, 30 (2009).

[56] M. Bashkanov PhD thesis, Univ. Tübingen 2006.

[57] A. Gardestig, G. Fäldt and C. Wilkin, Phys. Lett. B 421, 41 (1998).

[58] P. Adlarson et al., Eur. Phys. J. A 52, 147 (2016).

[59] M. Platonova and V. Kukulin, Phys. Rev. C 87, 025202 (2013).

[60] M. N. Platonova and V. I. Kukulin, Nucl. Phys. A 946, 117 (2016).

[61] R. Bhandari, R. A. Arndt, L. D. Roper and B. J. VerWest, Phys. Rev. Lett. 46, 1111 (1981).

[62] R. A. Arndt, I. Strakovsky, R. L. Workman and D. V. Bugg, Phys. Rev. C 48, 1926 (1993).

[63] R. A. Arndt, I. Strakovsky and R. L. Workman, Phys. Rev. C 50, 1796 (1994).

[64] C. H. Oh, R. A. Arndt, I. Strakovsky and R. L. Workman, Phys. Rev. C 56, 635 (1997).

[65] R. L. Shypit et al., Phys. Rev. Lett. 60, 901 (1988).

[66] R. L. Shypit et al., Phys. Rev. C 40, 2203 (1989).

[67] V. V. Anisovich, A. V. Sarantsev and D. V. Bugg, Nucl. Phys. A 537, 501 (1992).

[68] M. G. Ryskin and I. I. Strakovsky, Phys. Rev. Lett. 61, 2384 (1988).

[69] I. I. Strakovsky, Sov. J. Part. Nucl. 22, 296 (1991).

[70] N. Hoshizaki, Phys. Rev. C 45, R1414 (1992).

[71] N. Hoshizaki, Prog. Theor. Phys. 89, 245, ibid. 251, 563, 569 (1993).

[72] K. K. Seth, Proc. Baryon-Baryon Interaction and Dibaryonic Systems, Bad Honnef 1988, 41 (1988). 
[73] K. A. Olive et.al. (Particle Data Group), Chin. Phys. C 38, 090001 (2014).

[74] D. V. Bugg, Eur. Phys. J. A 50, 104 (2014).

[75] V. V. Sarantsev et al., Eur. Phys. J. A 43, 11 (2010).

[76] We note that this technique allows to be applied straight- forwardly also for the ${ }^{3} \mathrm{He}$ and ${ }^{4} \mathrm{He}$ cases by inclusion of the nucleons' motion according to the respective wavefunctions - see Refs. [12, 14]. 